\title{
Las coaliciones de partidos: ¿Un nuevo escenario para el sistema partidista chileno? ${ }^{1}$
}

Carlos HuneEus

\section{El problema: cambio y continuidad de coaliciones}

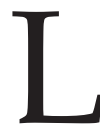

os procesos políticos en las nuevas democracias son dinámicos, por las complejidades del desmantelamiento de las instituciones y prácticas del autoritarismo y el establecimiento de las propias del orden pluralista. La consolidación democrática es un proceso complejo y dinámico, que conduce a diferentes niveles de institucionalización de los elementos del sistema político, que explica que ciertas generalizaciones formuladas a partir del examen de los primeros años después del cambio de régimen pierden vigencia con los resultados observados con posterioridad, que hacen necesario explicar los nuevos factores de poder que cobran mayor influencia. El grado de institucionalización del sistema político es heterogéneo, con desequilibrios entre sus organismos, con partidos que tienen mayor fortaleza organizativa que otros, algunos grupos de interés visiblemente dominantes y la existencia de discrepancias en la distribución del poder entre el Ejecutivo y el Congreso, pudiendo una rama ganar más influencia que la otra.

Las elecciones presidenciales y parlamentarias del 11 de diciembre del 2005 pueden ser vistas desde dos perspectivas distintas, aunque no con-

1. Trabajo preparado para ser presentado al Seminario "Elecciones chilenas 2005" organizado por el Departamento de Ciencia Política del Instituto de Asuntos Públicos, Universidad de Chile, 3 y 4 de noviembre 2005. 
tradictorias: como la cuarta contienda de la democratización de Chile, gatillada por el plebiscito sucesorio del 5 de octubre de 1988, o, por el contrario, como la primera de una nueva etapa de la política chilena, en la medida que el cuarto gobierno democrático, que probablemente será de la Concertación con Michelle Bachelet (PS), implicará un importante cambio, entre otras, por ser la primera mujer que llega a la presidencia. Sin embargo, ello también dependerá de las políticas que impulse la nueva administración de la Concertación por la Democracia, que se instalará el 11 de marzo del 2006 y que reúne a los partidos que estuvieron en la oposición al régimen militar del general Augusto Pinochet (19731990) y que ocuparon el centro y la izquierda hasta el golpe militar de 1973.

La continuidad de la Concertación responde a diversos factores institucionales e históricos, que apuntan en dirección a una cuarta administración controlada por ésta y no al comienzo de una nueva etapa porque la oposición hubiese logrado ganar los comicios presidenciales. Su origen se encuentra en el impacto de los conflictos entre el centro y la izquierda desde comienzos de los años 60, que llevaron a un proceso de polarización y confrontación que, en el contexto más amplio del conflicto político provocado por el gobierno de la Unidad Popular del presidente Salvador Allende, condujo al desplome de la democracia en 1973. En estas condiciones, surgió un régimen autoritario muy represivo, que se caracterizó, además, por la importante presencia de civiles en la dirección de los ministerios (Huneeus, 2000). Los altísimos costos humanos producidos por la dictadura provocaron un cambio en las opiniones políticas de la población a favor de una política de acuerdos y no de confrontación. Fue este cambio en la ciudadanía el que fortaleció el giro producido por los dirigentes políticos, que aprendieron las lecciones del pasado (Bermeo, 1992), favoreciendo una política consociativa, que se fue desarrollando a través de distintas instancias institucionales -el Grupo de Estudios Constitucionales en 1978; la Alianza Democrática en 1983 y la Concertación de Partidos por el No en 1988-, que dan cuenta de la solidez de esta coalición ${ }^{2}$. Esta legitimidad histórica constituye una poderosa base de sustentación de la Concertación, pues en esos largos años se han producido relaciones de confianza entre los dirigentes de los partidos que han ayudado a que se mantenga la cohesión de sus bancadas en el Congreso y a que se haya dado un apoyo muy decidido a cada uno de los presidentes.

Sin embargo, una historia de la Concertación apoyada en el desarrollo histórico del país, que es un pasado cada vez más lejano, plantea la pregunta sobre las perspectivas de esta coalición, y más específicamente hasta cuándo continuará. Una política de coalición entre partidos de centro e izquierda ha sido provechosa para el país, pero ha tenido altos costos para cada uno de ellos, en la medida que han debido compartir

2. No seguimos una explicación elitista de la democratización en Chile, que se ha usado en otros casos, como el de España. Véase por ejemplo Gunther (1992), pp. 38-80. 
las responsabilidades de gobierno. Este tipo de coalición amplia corresponde a una "gran coalición" de situaciones de emergencia que se dieron en las etapas posteriores al cambio de la dictadura a la democracia, pero termina cuando se ha consolidado el orden pluralista. Ahí están las experiencias de los primeros gobiernos de la postguerra en Europa o las coaliciones de los gobiernos del sur de Europa en las democratizaciones de la "tercera ola".

Por lo tanto, no son evidentes las justificaciones para la continuidad de la Concertación después de dos décadas, cuando el sistema político se encuentra plenamente establecido, existe un sistema múltiple de partidos y el orden económico tiene una alta legitimidad, mostrando un buen desempeño. Se sostiene que la alternancia de los gobiernos es indispensable para mantener el dinamismo del sistema político, lo cual apuntaría al fin de los gobiernos de la Concertación y a que la oposición alcanzara el poder. Sin embargo, esta necesidad es menos urgente en el caso chileno.

En primer lugar, la continuidad de los gobiernos de la Concertación ha sido positiva para lograr la consolidación del orden pluralista y ha sido un factor político de gran importancia en el momento de considerar las condiciones que han permitido un constante crecimiento económico. La discontinuidad de las políticas en el pasado por los cambios de presidente desde 1946 hasta el golpe de Estado de 1973 impidió que se mantuvieran a lo largo del tiempo las políticas de industrialización y crecimiento iniciadas a fines de los años 30 con el gobierno del Frente Popular del Presidente Pedro Aguirre Cerda (1938-1941) y de la Alianza Democrática del presidente Juan Antonio Ríos (1942-1946). Cada seis años hubo un nuevo presidente, con distintos ministros y nuevos programas para enfrentar la solución a los principales problemas del país. Los dirigentes de la Concertación valoran la continuidad como un medio para enfrentar los desafíos de superar el subdesarrollo.

En segundo lugar, porque ha habido una fuerte renovación de la elite dirigente con ocasión de cada presidente, lo cual ha moderado el impacto negativo que tiene la conservación del Poder Ejecutivo por el mismo partido durante un largo tiempo. Se teme, con bastante evidencia empírica, que en estas circunstancia no existan nuevas ideas impulsadas por sus altos funcionarios, que aplican los mismos planes y programas formulados cuando se ganaron las primeras elecciones. Se concluye que la única manera de lograr que haya ideas nuevas en los gobiernos sea la alternancia de los gobiernos. Sin embargo, en el caso de los tres gobiernos de la Concertación, cada presidente ha nombrado a nuevos ministros, subsecretarios, intendentes y gobernadores, por lo cual se ha logrado una gran renovación del personal directivo del Poder Ejecutivo y, con ello, nuevas ideas que han dado impulso a la labor del Ejecutivo.

Con todo, el resultado de las elecciones parlamentarias, con una caída del PDC en el Senado, que de 14 senadores baja a seis, y una derrota de varios diputados de éste ha producido un giro del eje de la Concertación 
hacia el bloque PS/PPD/PRSD con la ayuda del sistema binominal ${ }^{3}$, porque hay continuidad en votos. El hecho que el presidente sea de este sector por segunda vez plantea una compleja situación para el PDC, que buscará recuperar su poder e influencia en la coalición y enfatizar la necesidad de preparar una candidatura presidencial para el 2009. Esto puede crear tensiones al gobierno de Bachelet, que saldrá elegida con menos fuerza que la que tuvo cuando se impuso como una "candidatura ciudadana" en la izquierda y sobre el PDC.

\section{Cuadro 1}

Resultados electorales en la Cámara de Diputados, elecciones 2001 y 2005

\begin{tabular}{|c|c|c|c|c|c|c|c|c|}
\hline \multirow[t]{2}{*}{ Partido Político } & \multicolumn{4}{|c|}{2001} & \multicolumn{3}{|c|}{$2005^{*}$} & \multirow[b]{2}{*}{$\begin{array}{c}\% \\
\text { escaños }\end{array}$} \\
\hline & Votos & $\begin{array}{c}\% \\
\text { Votos }\end{array}$ & Escaños & $\begin{array}{l}\text { s } \% \\
\text { escaños }\end{array}$ & Votos & $\begin{array}{c}\% \\
\text { votos }\end{array}$ & Escaños & \\
\hline UDI & 1.547.209 & 25.18 & 31 & 25.8 & 1.456 .430 & 22.34 & 33 & 27.5 \\
\hline $\mathrm{RN}$ & 845.865 & 13.77 & 18 & 15.0 & 920.524 & 14.12 & 19 & 15.8 \\
\hline $\begin{array}{l}\text { Independientes } \\
\text { pro-Alianza }\end{array}$ & 327.121 & 5.32 & 8 & 6.6 & 145.604 & 2.23 & 2 & 1.6 \\
\hline PDC & 1.162 .210 & 18.92 & 23 & 19.1 & 1.354 .631 & 20.78 & 20 & 16.6 \\
\hline PPD & 782.333 & 12.73 & 20 & 16.6 & 1.006 .480 & 15.44 & 21 & 17.5 \\
\hline PS & 614.434 & 10.00 & 10 & 8.3 & 653.692 & 10.02 & 15 & 12.5 \\
\hline PRSD & 248.821 & 4.05 & 6 & 5.0 & 229.126 & 3.51 & 7 & 5.8 \\
\hline $\begin{array}{l}\text { Independiente } \\
\text { pro-Concertación }\end{array}$ & 135.191 & 2.20 & 3 & 2.5 & 130.936 & 2.00 & 2 & 1.6 \\
\hline Independientes $* * *$ & ** 90.439 & 1.48 & 1 & 0.8 & 138.071 & 2.1 & 1 & 0.8 \\
\hline $\begin{array}{l}\text { Izquierda extra- } \\
\text { parlamentaria** }\end{array}$ & 390.380 & 6.35 & - & - & 482.507 & 7.40 & - & - \\
\hline
\end{tabular}

Fuente: Sitio web del Ministerio del Interior: www.elecciones.gov.cl

* De acuerdo al 98,78\% de las mesas del país.

** Partido Comunista y Partido Humanista, que en la elección de 2001 no constituían pacto, por lo que se sumaron los porcentajes obtenidos por ambos.

*** Para la elección de 2001 incluye la votación obtenida por el Partido Liberal más los candidatos fuera de pacto. Para la elección de 2005, incluye a la Fuerza Regional Independiente y a los candidatos fuera de pacto. No refleja si después de ser electos ingresaron a algún partido.

\section{La continuidad política desde 1990}

Hay motivos para creer que la Concertación continuará en el futuro, sin perjuicio de los ajustes programáticos que sean necesarios. Su propio éxito constituye una fuerza centrípeta hacia su continuidad, mostrando una singularidad en las nuevas democracias presidenciales,

3. He analizado el sistema binominal en otro lugar, Huneeus (2005). 
pues no existe otro país en que la coalición gobernante haya logrado mantener el control del Poder Ejecutivo en forma continuada desde la primera elección democrática y en dos elecciones posteriores ${ }^{4}$, situación que es excepcional incluso en los regímenes parlamentarios en Europa (Engelmann, 1966: 260-283)5. Esto da cuenta del éxito de la gestión de la coalición gobernante, avalado por los buenos indicadores de desempeño económico, político y social. Este éxito, además, demuestra que el presidencialismo no es un orden político contrario a la consolidación de la democracia, al revés de lo predicho por Linz ${ }^{6}$ y Valenzuela (1985: 88-154) ${ }^{7}$ a fines de los años 80, quienes plantearon la necesidad de cambiar el régimen político a uno parlamentario.

\section{Cuadro 2}

\section{Comparación del desempeño de los gobiernos según variables macroeconómicas claves: 1974-2005}

\begin{tabular}{|c|c|c|c|c|c|}
\hline \multirow[b]{2}{*}{ Variable } & \multicolumn{5}{|c|}{ Durante el gobierno de: } \\
\hline & $\begin{array}{c}\text { Pinochet } \\
\text { 1974-89 }\end{array}$ & $\begin{array}{l}\text { Concertación } \\
\text { 1990-2005 }\end{array}$ & $\begin{array}{l}\text { Aylwin } \\
1990-93\end{array}$ & $\begin{array}{c}\text { Frei R-T. } \\
\text { 1994-99 }\end{array}$ & $\begin{array}{l}\text { Lagos } \\
2000-05\end{array}$ \\
\hline Crecimiento del PIB (\%) & 2,9 & 5,6 & 7,7 & 5,4 & 4,3 \\
\hline $\begin{array}{l}\text { Crecimiento de las } \\
\text { exportaciones }(\%)\end{array}$ & 10,7 & 8,5 & 9,6 & 9,7 & 6,5 \\
\hline Tasa de inflación ${ }^{b}$ & 79,9 & 7,8 & 17,7 & 6,1 & 2,9 \\
\hline Tasa de desempleo ${ }^{c}$ & 18,1 & $8,3^{\mathrm{d}}$ & 7,3 & 7,4 & $10,1^{\mathrm{d}}$ \\
\hline Salario real $(1970=100)$ & 81,9 & $122,3^{\mathrm{d}}$ & 99,8 & 123,4 & $138,9^{\mathrm{d}}$ \\
\hline Inversión bruta fija (\% del PIB)e & 15,3 & 23,8 & 21,0 & 25,4 & 24,5 \\
\hline $\begin{array}{l}\text { Superávit del gobierno general } \\
\text { (\% del PIB) }\end{array}$ & 0,3 & $0,8^{\mathrm{d}}$ & 1,6 & 1,1 & $0,0^{\mathrm{d}}$ \\
\hline Superávit estructural (\% del PIB) & & $0,7^{\mathrm{d}}$ & 0,4 & 0,8 & $0,8^{\mathrm{d}}$ \\
\hline
\end{tabular}

Fuentes: Tomado de Ffrench-Davis (2003, cuadro I.1) y actualizaciones de Ffrench-Davis. Basado en Banco Central de Chile y DIPRES. Las cifras de 2005 son muy provisionales.

a Tasas acumulativas anuales de crecimiento del PIB y exportaciones; promedio de tasas anuales en inflación y desempleo.

${ }^{\mathrm{b}}$ Diciembre a diciembre.

c Incluyen como desocupados a los trabajadores de los programas especiales de empleo; la cifra sin ellos es $13,3 \%$ en $1974-89,7,3 \%$ en $1994-99$ y $9,0 \%$ en $2000-04$.

d Cifras a 2004.

e En pesos de 1996.

4. El otro caso sería la República Checa, aunque el país se segregó de Checoslovaquia, lo que no constituye un hecho positivo. Destacan los casos de Chile y la República Checa. Véase Schmitter (1999), pp. 475-495. Hay casos de países en los cuales los segundos gobiernos democráticos han logrado mantenerse en el poder por tres elecciones consecutivas, como el Arena en El Salvador, pero se trató del principal partido opositor al primer gobierno democrático.

5. Nótese que la CDU/CSU en Alemania gobernó en solitario entre 1957 y 1961 porque obtuvo la mayoría absoluta.

6. Esta posición la expuso en diversos artículos Juan J. Linz (1990a), pp. 51-69; (1990b), pp. 84-91; (1994), pp. 3-87. Para el caso de Chile, véase a Valenzuela (1994), pp. 91-150.

7. Esta tesis fue actualizada en su contribución al libro que editó con Juan Linz (1994), pp. 91-150. 
Los tres gobiernos de la Concertación han impulsado una política de crecimiento con equidad que ha logrado una drástica reducción de la inflación, tasas moderadas de desempleo y altos niveles de crecimiento, en una estrategia de buscar reducir la pobreza y la extrema pobreza, lo que se ha conseguido (Ffrench-Davis, 2003). Han tenido una política de derechos humanos que apunta a verdad y justicia "en la medida de lo posible", como acertadamente lo definió el presidente Aylwin, y una subordinación gradual, pero efectiva, de los militares al poder civil ( $\mathrm{Hu}-$ neeus, 2000). La subordinación ha sido acompañada por el procesamiento por parte de los tribunales de justicia de un número significativo de oficiales y suboficiales por su participación en violaciones a los derechos humanos, lo que condujo, a su vez, a la condena y encarcelamiento, entre otros, del general Manuel Contreras, organizador y primer director de la DINA (Dirección de Inteligencia Nacional), principal órgano represivo de los primeros años de la dictadura (Huneeus, 2003: 169-188), y se expresó después en el procesamiento del general Humberto Gordon, director de la CNI. En esta dirección se encuentra el desafuero del general Pinochet por la Corte Suprema en agosto de 2000, posterior a su humillante detención en Londres el 16 de octubre de 1998 por orden de la justicia española, que lo mantuvo detenido durante quince meses en un suburbio de la capital británica. Esto ha sido acompañado por la ambiciosa reforma del sistema judicial, la que representa un valioso avance en la calidad de la democracia ${ }^{8}$.

El éxito de la democratización es alabado por políticos, altos funcionarios de organismos internacionales y analistas, siendo este proceso calificado como "modelo" (Drake y Jaksic, 1999). En un reciente seminario en España convocado para discutir la transición chilena, un cientista político alemán señalaba que:

...según el índice de transformación de Bertelsmann ${ }^{9}$, en 2003 Chile ocupaba el tercer lugar en América Latina de acuerdo al nivel de las transformaciones democráticas (superado solamente por Uruguay y Costa Rica). Además, se posicionaba en el primer lugar en relación al nivel de las transformaciones económicas y en el primer lugar respecto de la calidad de la gestión política. Comparando 116 países en el mundo, Chile se coloca entre los diez mejor evaluados en las tres dimensiones. Según el índice de libertad de Freedom House ${ }^{10}$, Chile ha mejorado de una evaluación de 2 en las dimensiones de derechos políticos y derechos cívicos en 1990, a una de 1 (lo máximo) en las dos dimensiones en 2005. Entre los países latinoamericanos, en 2005 solamente Costa Rica y Uruguay estaban en el mismo nivel en las dos dimensiones (Nolte, 2006).

A pesar de las transformaciones económicas, políticas y culturales producidas desde 1990, hay una notoria continuidad en las preferencias políticas de los chilenos, aunque esto no quiere decir inmovilismo, pues han ocurrido modificaciones. Hay estabilidad en la autoubicación en la

8. Sobre la reforma judicial, véase Correa (1999), pp. 281-315, y Blanco (2003), pp. 187-220.

9. Véase http: / / www.bertelsmann-transformation-index.de/

10. Véase http:/ / www.freedomhouse.org 
escala de izquierda a derecha, con una disminución de los que se ubican al centro y el aumento de los NS/NR, sin producirse el desplome del centro que ocurrió en España después de Franco, con la crisis y caída de la UCD (Huneeus, 1985), el partido que dirigió la transición, y el aumento de las posiciones a favor de la izquierda y la derecha. La inmensa mayoría de los chilenos se identifica en la escala de izquierda-derecha y la puntuación media está más a la derecha que en España (4,49 el 2003, mientras que en Chile era 5,55). Esta continuidad hace muy difícil que la oposición llegue a La Moneda en la medida que la Concertación siga siendo el arco iris del centro con la izquierda y se mantenga esa pluralidad, sin giros bruscos hacia la izquierda o hacia la derecha.

\section{Cuadro 3}

Autoposicionamiento en la escala política de izquierda a derecha, 1989-2005 P.: En política se habla normalmente de "izquierda y derecha". En una escala donde " 1 " es la izquierda y " 10 " es la derecha, ¿dónde se ubicaría usted?

\begin{tabular}{|c|c|c|c|c|}
\hline & Izquierda & Centro & Derecha & NS/NR \\
\hline Abr. 89 & 23 & 48 & 24 & 5 \\
\hline Abr. 90 & 26 & 48 & 20 & 6 \\
\hline Mar. 91 & 27 & 46 & 21 & 6 \\
\hline Mar. 92 & 22 & 47 & 20 & 11 \\
\hline Oct. 93 & 24 & 34 & 19 & 22 \\
\hline Abr. 94 & 30 & 38 & 16 & 15 \\
\hline Sep. 95 & 30 & 37 & 18 & 16 \\
\hline Dic. 95 & 26 & 39 & 17 & 17 \\
\hline Dic. 96 & 28 & 37 & 22 & 14 \\
\hline Dic. 97 & 27 & 39 & 24 & 10 \\
\hline Dic. 98 & 26 & 38 & 19 & 17 \\
\hline Sep. 99 & 26 & 36 & 25 & 12 \\
\hline Dic. 99 & 26 & 38 & 28 & 8 \\
\hline May. 00 & 23 & 37 & 24 & 14 \\
\hline Jul. 00 & 25 & 34 & 30 & 11 \\
\hline Sep. 00 & 22 & 40 & 27 & 11 \\
\hline Abr. 01 & 25 & 36 & 26 & 13 \\
\hline Jul. 01 & 23 & 35 & 25 & 17 \\
\hline Sep. 01 & 20 & 35 & 25 & 20 \\
\hline Dic. 01 & 22 & 38 & 22 & 18 \\
\hline Abr. 02 & 21 & 35 & 23 & 21 \\
\hline Jul. 02 & 18 & 32 & 29 & 21 \\
\hline Sep. 02 & 17 & 37 & 20 & 26 \\
\hline Dic. 02 & 19 & 26 & 26 & 29 \\
\hline Abr. 03 & 18 & 33 & 19 & 29 \\
\hline Jul. 03 & 19 & 25 & 30 & 26 \\
\hline Sep. 03 & 18 & 31 & 17 & 33 \\
\hline Dic. 03 & 20 & 32 & 19 & 29 \\
\hline Abr. 04 & 21 & 28 & 23 & 28 \\
\hline Jul. 04 & 18 & 30 & 27 & 25 \\
\hline Sep. 04 & 22 & 30 & 23 & 25 \\
\hline Dic. 04 & 23 & 27 & 23 & 26 \\
\hline Abr. 05 & 22 & 32 & 19 & 29 \\
\hline Jul. 05 & 24 & 33 & 19 & 24 \\
\hline
\end{tabular}

Fuente: BARÓMETRO CERC, julio de 2005. 


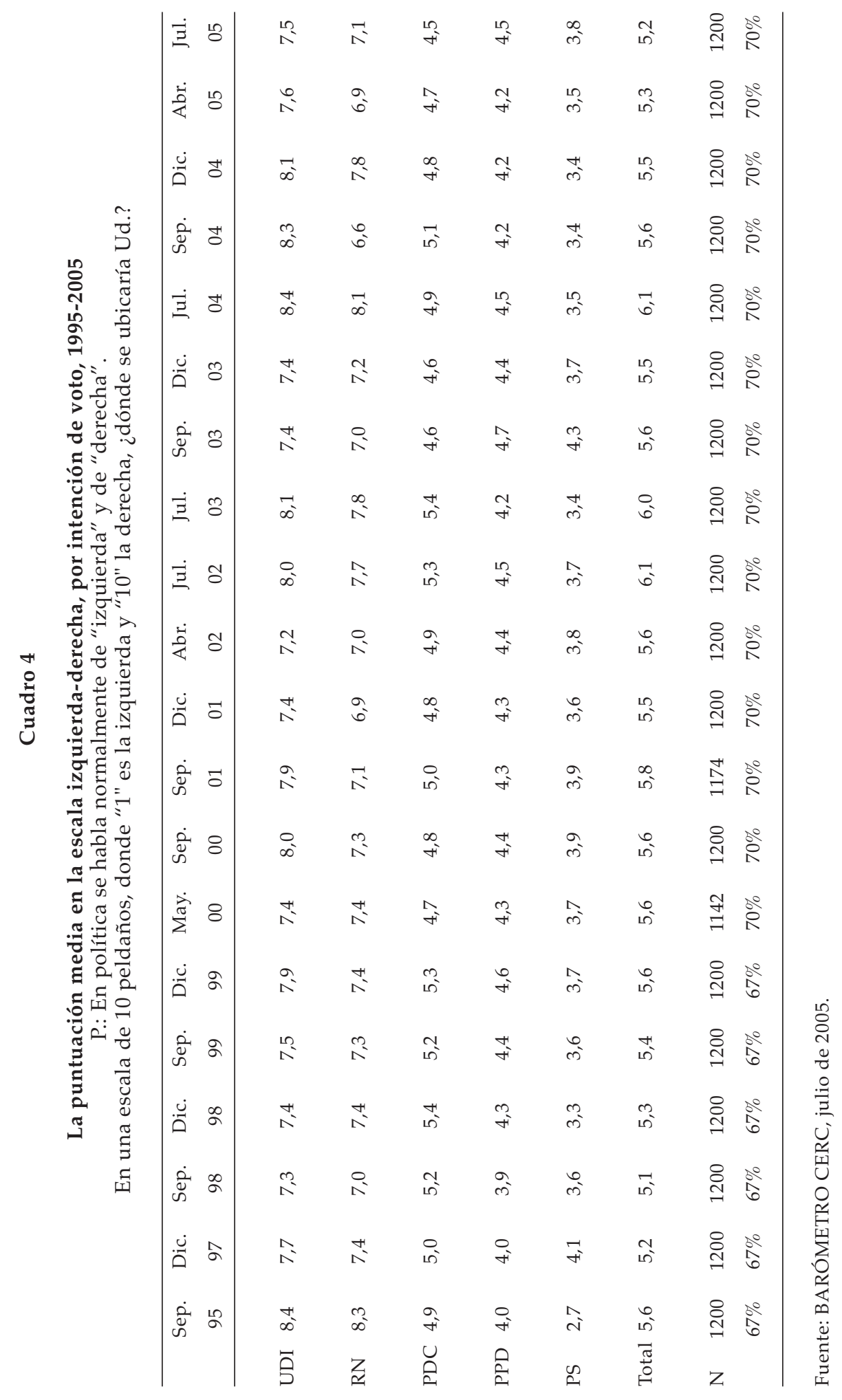


Sin embargo, cada nueva elección es más difícil y ésta lo está demostrando, a pesar de que se realiza en estupendas condiciones, con muy buenos indicadores económicos. De hecho, llama la atención que el excelente desempeño de los gobiernos de la Concertación no le haya permitido aumentar su apoyo electoral. Por el contrario, se produjo una fuerte caída de su votación en las elecciones parlamentarias de 1997, cuando existía una muy buena situación económica, y en la difícil elección de Ricardo Lagos (PS/ PPD) en las elecciones presidenciales de 1999. Recordemos que sólo 30.000 votos permitieron a Lagos superar al candidato de la derecha, Joaquín Lavín, teniendo que ir a una segunda elección en la cual se impuso nuevamente en forma muy estrecha. Esto demuestra dos cosas: por un lado, las limitaciones de la Concertación para captar nuevos votos como reconocimiento de su buen desempeño, lo cual constituye una diferencia con las experiencias de coaliciones exitosas en Europa después de la II Guerra Mundial, como la CDU/CSU y los liberales en Europa bajo el liderazgo de Konrad Adenauer (Schmidt, 2003). Por otro lado, ese electorado perdido, proveniente especialmente del PDC, fue captado por la derecha a través de la UDI, lo que le ha permitido expandir su tamaño, muy por encima del "tercio" que había tenido en el pasado. Esa pérdida se produjo en las elecciones parlamentarias de 1997, en los momentos de mejores indicadores económicos, lo cual muestra que el voto económico es de relativa capacidad explicativa para comprender los resultados electorales.

\section{La derecha después de Lavín}

La posibilidad que ésta sea la elección que abre una nueva etapa del desarrollo político de Chile no sólo depende de las decisiones que adopten los dirigentes de los partidos de la Concertación, sino también de las que tomen los de la Alianza por Chile. Éstos se encuentran ante la inminente cuarta derrota consecutiva, que los dejaría veinte años fuera del gobierno y con un complicado futuro, como consecuencia de las heridas producidas por la dura competencia electoral.

En efecto, hubo una intensa confrontación por parte de Sebastián Piñera contra Joaquín Lavín con la finalidad de derrotar a la UDI y pasar a segunda vuelta, aplastando así a la UDI para debilitarla como partido dominante en la derecha y como partido mayoritario del país. Consiguió lo primero, porque superó a Lavín por $25,41 \%$ a $23,2 \%$ de los votos, pero no lo segundo porque la UDI logró un apoyo electoral similar al de Lavín. Sus postulantes a la Cámara alcanzaron un 22,34\%, mientras que los de RN lograron menos de diez puntos que Piñera, 14,12\%. RN perdió las dos elecciones senatoriales en Santiago, imponiéndose Pablo Longueira contra Lily Pérez en Santiago oriente, y en la otra, Jovino Novoa contra Roberto Fantuzzi. Esto demuestra que la popularidad de Piñera no se traspasó a sus candidatos. Por otro lado, su estrategia de abrirse al centro para ganar votos que eran de la Concertación para ampliar la base 
de apoyo de la Alianza que le permitieran derrotar a la Concertación en la segunda vuelta, fue llevada a cabo a través de una confrontación con el PDC. Buscó la adhesión de personas de este partido que lo apoyaran y se declaró como el genuino representante del humanismo cristiano, descalificando a Michelle Bachelet (PS), la abanderada de la coalición de gobierno, como agnóstica. La DC vio esta táctica como una manera de dividirla, recordando las iniciativas hechas en esa dirección por la izquierda a finales de los años 60 y comienzos de los 70, que llevaron a dos rupturas del partido, que lo debilitaron, dejando una memoria a favor de la cohesión y de rechazo a los ataques externos. Si Piñera no gana la segunda vuelta, quedará debilitado porque su partido es más débil que antes y la UDI está fortalecida, con un sólido liderazgo de Lavín, pues éste podrá mostrar haber ayudado al triunfo de su partido.

La opción política adoptada por "el gremialismo" bajo el liderazgo de Joaquín Lavín fue de grandes dimensiones, pues significó un nuevo estilo de hacer política -el "cosismo", es decir, hacer "cosas concretas, sin contenido político y que preocupan a la gente-, el cual le reportó enormes ventajas electorales. Lo llevó a las puertas de La Moneda en 1999 y permitió al "gremialismo" convertirse en el principal partido de Chile en las elecciones parlamentarias de 2001. Se caracterizó por un discurso que buscó despolitizar la acción pública para dejar de lado el peso de su vinculación con el régimen militar; priorizó atender las necesidades materiales inmediatas de la gente, privilegió las acciones "ejecutivas" y puso una atención especial al empleo de la propaganda política para ganar popularidad. Una nueva derrota pone en tela de juicio la vigencia de ese discurso. ¿Qué ideas matrices lo reemplazarán? ¿Qué personalidades tratarán de ocupar el lugar que Lavín tuvo en estos años luego de esta segunda derrota presidencial o seguirá siendo Lavín el líder de la derecha, como Salvador Allende lo fue de la izquierda durante un largo tiempo?

El desequilibrio en el grado de institucionalización entre RN y la UDI provoca tendencias centrífugas que dificultan la definición de una estrategia común. Puesto que la UDI es un partido con un alto grado de institucionalización, con dirigentes cohesionados por lazos históricos y personales muy sólidos, puede enfrentar con mejores condiciones las decisiones que deberá adoptar el sector. La cohesión también puede ser un factor perturbador, en la medida que sus dirigentes se han acostumbrado a actuar en solitario, sin considerar seriamente los intereses de sus socios de $\mathrm{RN}$, lo cual se traduce en dificultades para seguir una política de coalición, que provoca desconfianzas en RN. Sin embargo, la UDI tiene una organización fuerte y dirigentes a lo largo del país. Tuvo una plantilla parlamentaria de mejor calidad que RN, lo que explica haberse impuesto claramente sobre $\mathrm{RN}$, especialmente en las dos elecciones senatoriales de Santiago: el diputado Víctor Pérez le arrebató el cupo al senador Mario Ríos en la VIII interior y Cristián Leay estuvo a escasos 800 votos de hacer lo mismo con el senador Carlos Cantero, vicepresidente de RN, en la II Región. Las elecciones del 11 de diciembre confir- 
man la hegemonía de la UDI en la derecha y Piñera queda con un partido debilitado y rechazado por el PDC.

Este posible escenario político planteará tensiones, por la tradición de conflictos entre Piñera y la UDI en los últimos años y por la intensa competencia electoral en los comicios presidenciales y parlamentarios del 2005, que requerirá enormes esfuerzos para restablecer la unidad del sector. Lo más probable es que después de los comicios, el bipartidismo de la derecha sea una realidad dominante en el sector, con acuerdos a nivel parlamentario en temas específicos, pero cada partido aspirará a presentar un candidato presidencial en cuatro años más. La competencia en la derecha no dejará de tener consecuencias en la política de la Concertación, en la medida que la estrecha mayoría que tendrá en la nueva legislatura le permitirá buscar acuerdos con uno u otro partido y dejar de lado el desarrollo político desde 1990 dominado por el antagonismo entre dos bloques cohesionados. Como ésta será la segunda ocasión que se produce una segunda vuelta y los partidos de la Concertación mantienen el control del gobierno, aunque con menos votos que en la anterior contienda presidencial, el PDC tendrá nuevos argumentos para levantar una candidatura presidencial en cuatro años más y que la Concertación concurra a ella con dos candidatos, cada uno de los cuales impulsará la respectiva lista parlamentaria. Cuando no hay una derecha que aparece con menos capacidad de ganar la presidencia por su división, se desvanecen los motivos para buscar una candidatura común.

En la medida que la UDI y RN privilegien la continuidad de la política de alianza, ello constituirá un factor de estímulo para que la Concertación se prolongue como coalición. Sin embargo, ello dependerá de las estrategias que sigan la UDI y RN en el cuarto gobierno democrático, lo cual, a su vez, dependerá del desenlace presidencial y parlamentario del 2005. La coincidencia en diversas políticas económicas de los expertos de los tres principales partidos de la coalición oficialista -PS, PPD y PDC- y de la oposición, podría favorecer acuerdos en torno a políticas que den más credibilidad a un posible gobierno de la oposición en las elecciones del 2009. No es inimaginable que la UDI, luego de confirmarse como el principal partido de Chile y como una manera de aplastar a Piñera, esté dispuesta a llegar a acuerdos con el gobierno sobre temas controvertidos, como por ejemplo el sistema binominal, para seguir creciendo a costa de RN y buscar votos centristas.

El problema es si acaso habrá una continuidad de la competencia electoral entre los dos bloques, iniciada en 1989 por tiempo indeterminado o, por el contrario, empezará una competencia entre los partidos, que dará posibilidad a que se formen nuevas alianzas, superando la alineación de los actuales bloques. Esta variedad de coaliciones es propia de las democracias avanzadas con sistemas múltiples de partidos, de las "democracias de consenso", según la tipología de Lijphart (2000). No sólo el PDC puede mirar hacia RN para buscar entendimientos, sino también el PPD y el propio PS, aunque no se debe excluir el protagonismo de la UDI, 
que cuenta con un partido con un fuerte grupo parlamentario y un liderazgo muy decidido. La flexibilidad futura de las coaliciones tiene que ser provocada por los partidos de oposición, que son los que tienen la necesidad de tomar decisiones radicales para acercarse al poder.

\section{La difícil vida de los partidos de gobierno en el presidencialismo}

La continuidad de la Concertación se ve dificultada por los altos costos que tiene mantenerse en el gobierno desde 1990 y porque el sistema institucional no la favorece. El presidencialismo tiene una difícil relación con los partidos, con ministros y subsecretarios que reconocen militancia de partidos, pero que los ignoran y hasta cultivan un estilo contestatario hacia ellos. Los ministros son reclutados entre profesionales, no siempre militantes de partido, y si lo son, no han tenido vida partidaria. Este reclutamiento es fuente de problemas para los partidos, pues estos funcionarios desconocen las necesidades de las organizaciones, no están al tanto de cuáles son los más adecuados dirigentes intermedios y superiores y, al no ser parlamentarios, carecen de conocimiento sobre las necesidades que plantea la lucha política y electoral. La primacía asignada a las políticas económicas centraliza las decisiones en el Ministerio de Hacienda, en el cual predominan criterios a veces tecnocráticos de escasa significación para los dirigentes de los partidos y aún menos comprensibles para los ciudadanos.

El énfasis en las políticas públicas con criterios "técnicos" y el rechazo a una mirada política sobre ellas han conjurado en contra de una mayor visibilidad de los importantes cambios institucionales hechos al sistema económico existente cuando se inauguró la democracia. No sólo se ha enfatizado una política social para buscar mejores condiciones de equidad con criterios distintos al neoliberalismo que inspira a los técnicos de la derecha, sino que se han fortalecido instituciones y procesos económicos ajenos al neoliberalismo, como la protección al consumidor y al medio ambiente con la creación del Servicio Nacional del Consumidor (SERNAC) y la Comisión Nacional del Medio Ambiente (CONAMA), respectivamente. Ha habido una mayor regulación de áreas privatizadas durante el régimen autoritario, que disminuyen la discrecionalidad de los empresarios. Algunos de estos cambios son de escasa espectacularidad, como ocurre con la transparencia en las votaciones de las AFP en las juntas de accionistas de las empresas en las que tienen acciones, pero son de enorme relevancia para definir el gobierno de las empresas. Esta falta de discurso político en el sector económico ha influido en planteamientos a favor de "cambiar el modelo" por personalidades que van desde la izquierda extraparlamentaria hasta el mundo de los negocios.

Las relaciones de los ministros con los parlamentarios suelen verse afectadas por un bajo nivel de confianza. Los primeros tienden a desconfiar de las demandas de éstos, considerándolas iniciativas destinadas a apoyar intereses específicos, generalmente contrapuestos con el cumpli- 
miento del programa de gobierno. Las declaraciones del ministro de Hacienda, Nicolás Eyzaguirre (PPD) en la ENADE 2002 de que "los políticos son atroces, sólo los dictadores son peores", reiteradas el año 2003, cuando acusó a los parlamentarios oficialistas de "bajarse los pantalones" ante las demandas de la Agrupación Nacional de Empleados Fiscales (ANEF) son ilustrativas de este estilo que no favorece las relaciones entre ambos poderes del Estado ni la imagen de los políticos.

Los parlamentarios, por su parte, tienen reservas hacia los ministros, considerándolos rígidos tecnócratas que deciden de acuerdo a criterios alejados de las necesidades reales de los ciudadanos. El descuido en las políticas hacia los trabajadores y los sindicatos por parte de las autoridades de gobierno en los últimos años, ha dañado a las organizaciones de los trabajadores, mermando su poder político, lo que repercute en la imagen de desigualdad que predomina en el país. La despreocupación de los partidos oficialistas por el mundo sindical, con una Central Unitaria de Trabajadores (CUT) presidida por un militante de la Concertación, Antonio Martínez (PS), ha debilitado también a las organizaciones de trabajadores.

La percepción ciudadana sobre las desigualdades y discriminaciones da cuenta de una crítica a la gestión económica y política, pues no se refiere sólo a las desigualdades de ingreso, sino también a las discriminaciones de tipo social. Esta percepción subjetiva, crítica del sistema económico y político, debiera provocar cambios en las políticas y en los estilos de implementación de ellas para corregir esa situación, porque esa imagen daña la credibilidad de la coalición gobernante y favorece alternativas de izquierda, hoy bloqueadas por el sistema binominal.

Pese a la menor visibilidad de los partidos de gobierno, ellos mantienen un rol fundamental en el sistema político y la ciudadanía los sigue considerando como instituciones fundamentales. Son ubicados en la escala de izquierda-derecha, cada uno en su lugar: el PS en la izquierda, el PPD en el centro-izquierda, el PDC al centro, habiendo una distancia promedio en torno a 1 punto. Los partidos son ubicados entre el 3.01 y el 5.50 en julio del 2004.

Esta es una gran diferencia con los partidos de la derecha, UDI y RN, que son ubicados prácticamente en el mismo lugar, entre el 8 y el 9, habiendo menos de un punto de distancia entre ambos. Los dos partidos apelan a su electorado desde una perspectiva restringida, que limita su acción, mientras que la Concertación lo hace desde un abanico más amplio, que da cuenta del arco iris de la coalición gobernante, uno de sus principales factores de poder.

Sin embargo, las campañas electorales son impulsadas dando una imagen de distancia con los partidos, sin usar sus símbolos e indicar sus nombres, a diferencia de lo que ocurre en los regímenes parlamentarios en que es normal dar el nombre del partido. Esta tendencia a la personalización de las campañas choca con la realidad que comienza en la noche 
de la elección, cuando los presidentes de los partidos dan cuenta de sus triunfos o de sus derrotas. Y también choca con la realidad de que el trabajo político en las democracias se hace con ellos y no sólo con criterios de personalización.

\section{Cuadro 5}

Ubicación de los partidos en el eje izquierda-derecha (puntuación media)

P.: También los partidos políticos se ubican normalmente de "izquierda"

a "derecha". En una escala de 10 peldaños, siendo 1 la "izquierda" y 10 la "derecha", dónde ubicaría Ud. a los siguientes partidos:

\begin{tabular}{lcccccccccccccccc}
\hline & \multicolumn{2}{c}{ Jun. 96 } & \multicolumn{2}{c}{ Jun. 97} & \multicolumn{2}{c}{ Jun. 98 } & \multicolumn{2}{c}{ Jul. 99 } & \multicolumn{2}{c}{ Jul. 00 } & \multicolumn{2}{c}{ Jul. 02 } & \multicolumn{2}{c}{ Jul. 04 } \\
& Media & D.st. & Media & D.st. & Media & D.st. & Media & D.st. & Media & D.st. & Media & D.st. & Media & D.st. \\
\hline UDI & 8.55 & 1.95 & 8.65 & 1.93 & 8.87 & 1.88 & 8.88 & 1.75 & 8.67 & 2.01 & 7.99 & 1.93 & 9.18 & 1.37 \\
RN & 8.23 & 1.89 & 8.17 & 1.80 & 8.48 & 1.80 & 8.49 & 1.69 & 8.46 & 1.92 & 7.74 & 1.75 & 8.67 & 1.51 \\
PDC & 5.35 & 1.74 & 5.59 & 1.72 & 5.77 & 1.67 & 5.33 & 1.72 & 5.4 & 1.51 & 5.26 & 1.59 & 5.54 & 1.56 \\
PPD & 4.37 & 1.87 & 4.14 & 1.61 & 4.25 & 1.87 & 3.89 & 1.69 & 4.11 & 1.55 & 4.46 & 1.50 & 4.12 & 1.67 \\
PS & 3.02 & 1.84 & 3.0 & 1.67 & 3.01 & 1.84 & 2.74 & 1.56 & 2.87 & 1.48 & 3.71 & 1.89 & 3.11 & 1.52 \\
PC & 1.80 & 1.66 & 1.73 & 1.50 & 1.81 & 1.78 & 2.74 & 1.56 & 1.56 & 1.17 & 2.89 & 1.98 & 1.48 & 1.08 \\
N & 1240 & & 1200 & 1200 & & 1200 & & 1200 & & 1200 & & 1200 & \\
& $67 \%$ & & $67 \%$ & & $67 \%$ & & $67 \%$ & & $70 \%$ & & $70 \%$ & & $70 \%$ & \\
\hline
\end{tabular}

Fuente: BARÓMETRO CERC, julio de 2004.

\section{La práctica de cooperación en el Congreso}

El futuro de las coaliciones no puede examinarse sólo en relación al gobierno y quien lo preside, sino también considerando el trabajo parlamentario (Huneeus y Berrios, 2004). Esto ha sido algo ignorado en la literatura sobre el presidencialismo. De hecho, una de las arenas institucionales de la política de coaliciones es el Congreso, en el que los parlamentarios actúan siguiendo las decisiones de su respectiva bancada y se coordinan con las otras de la misma coalición. El Congreso, especialmente el Senado, ha ganado autoridad y poder en el sistema político, especialmente con las facultades de nominación de altos funcionarios del Estado que ha adquirido la Cámara Alta, que incluye ministros y fiscales de la Corte Suprema. Si bien es cierto que el presidencialismo chileno tiene una fuerte centralidad en el jefe de Estado, ésta ha sido morigerada desde el liderazgo del presidente Patricio Aylwin que dio un amplio espacio de acción a los parlamentarios, mantenido por sus sucesores.

El presidente no actúa solo, sino que tiene que entenderse con el Congreso: hasta ahora ello ha sido indispensable porque no ha tenido mayoría en el Senado por la presencia de los senadores designados, la mayoría de los cuales han votado con la oposición. Desde el 2006, el presidente tendrá mayoría en el Senado por la victoria de la Concertación en el distrito de la VIII costa, doblando con Alejandro Navarro (PS) y Hosaín 
Sabag (PDC), hecho inédito en la historia republicana, pues el sistema de elección parcial del Senado impidió a todos los presidentes tener mayoría en la Cámara Alta. Esto planteará un nuevo escenario, en que la Concertación no podrá atribuir a la oposición la imposibilidad de sacar algunos proyectos de leyes, por ejemplo, más derechos a los trabajadores, protección al medio ambiente y otros.

El presidente, además, no puede gobernar sin leyes, pues sus principales policies requieren una ley que las autorice y les dé financiamiento (Rose, 1978). El presidente no define sólo la agenda legislativa; tiene que considerar los intereses de los parlamentarios oficialistas y de la oposición. Incorpora aquellos proyectos de ley que tienen un claro respaldo en los parlamentarios de sus bancadas y deja de lado aquéllos que no lo tienen. Los parlamentarios pueden cambiar las prioridades del Ejecutivo que acepta legislar sobre temas que había rechazado antes, como ocurrió con el royalty, pues el presidente Lagos se había comprometido en la campaña electoral de 1999 a no subir los impuestos.

El Congreso no es una institución marginal al proceso político y tampoco es estático, sino que ha tenido un amplio e importante espacio de participación en el proceso político, que se ha expandido desde la inauguración de la democracia. Los parlamentarios tienen una estrecha relación con los de su bancada y con los de su coalición, pero también son positivas las relaciones con los de otra coalición (cuadro 5).

\section{Cuadro 6}

Relaciones de colaboración entre diputados y otros organismos, grupos e instituciones, congresos de Chile y Argentina

P. ¿Cómo definiría Ud. las relaciones que mantiene, durante su trabajo diario, con ...? Sólo respuesta "muy colaborativa"

\begin{tabular}{|c|c|c|c|c|c|c|c|c|c|c|}
\hline & \multicolumn{6}{|c|}{ CHILE $^{*}$} & \multicolumn{4}{|c|}{ Argentina** } \\
\hline & UDI & RN & PDC & PPD & PS & $\begin{array}{l}\text { Total } \\
\text { Chile J }\end{array}$ & $\begin{array}{l}\text { Partido } \\
\text { usticialista }\end{array}$ & UCR & Otros & $\begin{array}{c}\text { Total } \\
\text { Argentina }\end{array}$ \\
\hline $\begin{array}{l}\text { Autoridades } \\
\text { de gobierno }\end{array}$ & 15 & 30 & 46 & 21 & 44 & 35 & 16 & 5 & 14 & 13 \\
\hline $\begin{array}{l}\text { Diputados de } \\
\text { su partido }\end{array}$ & 85 & 65 & 51 & 21 & 50 & 58 & 38 & 85 & 70 & 59 \\
\hline $\begin{array}{l}\text { Diputados de } \\
\text { otros partidos / } \\
\text { otros bloques }\end{array}$ & 12 & 25 & 21 & 7 & 13 & 17 & 10 & 25 & 19 & 16 \\
\hline $\begin{array}{l}\text { Diputados de } \\
\text { su coalición }\end{array}$ & 27 & 40 & 28 & 21 & 50 & 33 & - & - & - & - \\
\hline $\begin{array}{l}\text { Diputados de } \\
\text { otra coalición } \\
\text { Autoridades del } \\
\text { gobierno provincial } \\
\text { y/o municipal }{ }^{* * *}\end{array}$ & $\begin{array}{l}4 \\
1 \\
-\end{array}$ & 25 & 15 & 7 & 13 & 13 & - & - & - & - \\
\hline
\end{tabular}

Fuente: *Encuesta diputados y ex diputados Chile, 2004. ${ }^{* *}$ Encuesta diputados Argentina 2005. 
Más allá de las disposiciones constitucionales que sorprenden a los lectores de la Constitución de 1980, disminuidas por las reformas constitucionales de 1989 y 2005, en la práctica el presidente no es un actor que domina de sobremanera la marcha del régimen político y tiene que entenderse con el Legislativo. El caso de Chile, como se ha argumentado para Argentina ${ }^{11}$ y Brasil ${ }^{12}$, demuestra que el desarrollo institucional de la democracia apunta hacia una mayor visibilidad del rol del Congreso y, por ello, a promover una política de negociaciones y compromisos al interior de la coalición gobernante y con parlamentarios de la oposición. Esto pone de manifiesto la importancia de los partidos, que han tenido un valioso rol en el Congreso y sirven de comunicación entre éste y la sociedad, y entre aquél y el Ejecutivo.

No se puede inferir que este mayor protagonismo del Congreso constituye una demostración del cambio hacia el parlamentarismo, ni tampoco significa que el presidencialismo funciona como uno de tipo parlamentario, como afirman Colomer y Negretto (2005: 60-89). Ambas conclusiones comparten una mirada pesimista del presidencialismo y optimista del parlamentarismo, considerado como el modelo ideal para organizar una democracia en América Latina y que debiera ser importado. El caso de Chile desde 1990 -aunque también hubo períodos buenos antes de 1973desmiente las generalizaciones sobre la inviabilidad del presidencialismo en América Latina y sobre la necesidad del cambio de la forma de gobierno hacia uno parlamentario. Hasta ahora el presidencialismo se ha mirado sólo en torno al presidente y sus facultades legislativas que dominan al Congreso, como lo hicieron Shugart y Carey (1992), pero la realidad es más compleja y cambiante.

\section{¿Flexibilización de las coaliciones? Competencia presidencial y parla- mentaria}

Hemos preguntado hasta cuándo Chile seguirá con una política dominada por dos grandes coaliciones y cuándo se producirá la apertura a nuevas formas de cooperación entre los partidos. Los cambios políticos económicos, sociales y culturales ocurridos en el país en los últimos años plantean enormes desafíos a los dirigentes de los partidos de la Concertación que los empujan a continuar el camino iniciado hace más de un cuarto de siglo, cuando en 1978 se creó el Grupo de Estudios Constitucionales, el Grupo de los 24. Es posible la continuidad, pero requiere una alta dosis de renovación y cambio que supone no sólo definir políticas en esa dirección, sino también abrir nuevas formas de cooperación entre los partidos.

11. Remito a los artículos de Mustapic, (2000: 577); Llanos, (2001: 67-99); Llanos y Margheritis, (1999: 441-475).

12. Un minucioso e interesante examen de la amplia bibliografía brasileña sobre el sistema político en el Brasil hace Palermo (2000). Véase también Figueiredo y Linomngi (2003). Véase también a Lamounier (2003). 
La competencia presidencial de la derecha constituye un primer paso hacia una flexibilización de las coaliciones, que repercutirá en la Concertación, pues si las elecciones no se definen en primera vuelta, porque la derecha va dividida, no hay motivos para que cada partido no lleve un abanderado presidencial para que conduzca la campaña parlamentaria. La primera vuelta es para elegir al Congreso, y la segunda, al presidente. Se puede acordar una competencia civilizada, como de hecho se está dando en la parlamentaria. La competencia es fundamental para la vitalidad de los partidos.

La oposición no tiene la misma serenidad que las colectividades de gobierno, porque el temor al aislamiento es demasiado fuerte, y ante ello prevalecerán probablemente los argumentos a favor de seguir unidos. $\mathrm{Si}$ la derecha lo hace, eso empujará a la Concertación a seguir un camino similar. Sin embargo, la aparición de la candidatura de Tomás Hirsch y la coalición Juntos Podemos Más puede forzar a la Concertación a flexibilizar su política de alianzas. Para enfrentar la segunda vuelta, la Concertación y el propio gobierno buscaron eliminar el sistema binominal con un proyecto en la Cámara que no prosperó porque la derecha se abstuvo. Hay ahora mayor fuerza a favor del cambio electoral porque el PDC ha salido perjudicado y porque el sistema de partidos en general se ve perjudicado por sus efectos perturbadores en la competencia electoral entre colectividades aliadas y no entre las adversarias.

La eliminación del sistema binominal es indispensable para que se cuente con un adecuado sistema de representación. Hasta ahora, la Concertación ha impuesto a la derecha la aprobación de numerosas leyes a las que ésta se resistió durante años, pero no ha puesto similar energía en la eliminación de este enclave autoritario. Ello se explica por el conflicto de intereses que hay en ello, porque la eliminación del sistema binominal tendría costos para el pacto PS/PPD, pues perdería votos hacia la izquierda que hasta ahora ha tenido por el efecto del voto útil en esos electores, los cuales apoyarían a los candidatos del PC o de otra colectividad si tuviesen posibilidades reales de ser elegidos. La exclusión del Partido Comunista y la fuerte barrera a nuevos partidos que articulen demandas no satisfechas por los partidos existentes -como la demanda ecológica, que ha sido descuidada por los partidos- tienen altos costos para la democracia, que dañan a las propias colectividades oficialistas. Las restricciones a la competencia electoral entre los propios partidos de la Concertación -el PS no compite con el PPD- debilitan a los propios partidos, porque ninguno de ellos puede desarrollar a nivel nacional una organización electoral propia.

En consecuencia, las elecciones del 11 de diciembre del 2005 conducirán a un cuarto gobierno de la Concertación, lo cual significará la continuidad de una política de coaliciones que ha habido en las últimas décadas. También significará el comienzo de una nueva política entre el PDC y el PS/PPD que puede conducir a un cambio en la política de coaliciones de cara a las elecciones del 2009. La alta votación que obtu- 
vo la oposición con sus dos candidatos, superando a Michelle Bachelet, no es un hecho menor, que plantea un complejo escenario para las próximas elecciones presidenciales. La estrecha mayoría que obtuvo la Concertación en el Senado obligará al gobierno a continuar en la política de compromisos con la oposición que ha llevado desde 1990, como ocurrió en el primer gobierno democrático. La división de la derecha favorece esa tendencia. Esta puede ser apoyada tanto por el PS/PPD como por el PDC, que puede tener viabilidad en acuerdo con RN. La UDI quedaría en ese escenario aislada, pero es un partido que tiene capacidad de iniciativa política, porque tendrá un buen grupo de parlamentarios. Esta política será compleja de implementar por las tendencias centrífugas que pueden aparecer en la Concertación como consecuencia del triunfo del bloque PS/PPD/PRSD sobre el PDC. En síntesis, el cuarto gobierno democrático puede ser uno de transición hacia una nueva política de coaliciones.

\section{Bibliografía}

Bermeo, Nancy. 1992. "Democracy and the Lessons of Dictatorship". Comparative Politics 24 (3): 273-291.

Blanco, Rafael. 2003. "El programa de justicia del Gobierno de Eduardo Frei". En: Muñoz, Óscar y Stefoni, Carolina (eds.). El Período del Presidente Frei RuizTagle. Santiago, FLACSO-Editorial Universitaria: 187-220.

Colomer, Joseph y Negretto, Gabriel. Enero 2005. "Can Presidentialism work like Parliamentarism?". Government and Opposition 40 (1): 60-89.

Correa, Jorge. 1999. "Cenicienta se queda en la fiesta. El poder judicial chileno en la década de los 90". En: Drake, Paul y Jaksic, Iván (eds.). El modelo chileno. Democracia y desarrollo en los noventa. Santiago: LOM Ediciones: 281-315.

Drake, Paul y Jaksic, Iván (eds.). 1999. El modelo chileno. Democracia y desarrollo en los noventa. Santiago: LOM Ediciones.

Engelmann, Frederick C. 1966. "Austria: The Polling of Opposition". En: Dahl, Robert A., Political Opposition in Western Democracias. New Haven, Yale University Press: $260-283$.

Ffrench-Davis, Ricardo. 2003. Entre el neoliberalismo y el crecimiento con equidad. Tres décadas de política económica en Chile. Santiago: J.C. Sáez Editor ( $3^{a}$ edición actualizada).

Figueiredo, Argelina Cheibub y Limongi, Fernando. 2003. "Congress and Decisión-Making in Democratic Brazil". En: Kinzo, Maria D'Alva y Dunkeley, James (eds.). Brazil since 1985: Politics, Economics and Society. Londres, Institute of Latin American Studies: 62-83.

Figueiredo, Argelina Cheibub et al. 2000. "Presidential Power, Legislative Organizations, and Party Behaviour in Brazil". Comparative Politics 32 (2): 151-170.

Gunther, Richard. 1992. "Spain: the very model of the modern elite settlement". En: Higley, John, Gunther, Richard \& Burton Michael G. (comp.). Elites and Democratic Consolidation in Latin America and Southern Europe. Cambridge, Cambridge University Press: 38-80. 
Huneeus, Carlos. 1985. La Unión de Centro Democrático y la transición a la democracia en España. Madrid: Centro de Investigaciones Sociológicas-Siglo XXI Editores.

Huneeus, Carlos. 2000. El régimen de Pinochet. Santiago: Editorial Sudamericana.

Huneeus, Carlos. 2003. "The consequences of the Pinochet case for Chilean politics". En: Davis, Madeleine (ed.) The Pinochet Case. Origins, Progress and Implications. Londres, Institute of Latin American Studies: 169-188.

Huneeus, Carlos. 2005. “Chile: A System frozen by Elite Interests". En: International IDEA, Electoral System Design: The New International IDEA Handbook. Estocolmo: IDEA: 78-81.

Huneeus, Carlos y Díaz de Valdés, Luz María. “Presidencialismo y partidos políticos. La experiencia del gobierno de Jorge Alessandri (1958-1964)", ponencia presentada a las XVI Jornadas de Historia, 18-19 noviembre 2003.

Huneeus, Carlos y Berríos, Fabiola. 2004. “El Congreso Nacional en un régimen presidencial. El caso de Chile". Revista de Derecho Público 66: 61-96.

Lamounier, Bolívar. 2003. "Brazil: An Assessment of the Cardozo Administration". En: Domínguez, Jorge I. y Shifter, Michael (eds.). Constructing Democratic Governance in Latin America. Baltimore, The Johns Hopkins University Press: 269-291.

Llanos, Mariana. Febrero 2001. “Understanding Presidential Power in Argentina: a Study of the Policy of Privatization in the 1990s". Journal of Latin American Studies 33 (1): 67-99.

Llanos, Mariana y Margheritis, Ana. 1999. “Liderazgo presidencial y dinámica institucional durante el primer período presidencial de Menem. El caso de las privatizaciones". Política y Gobierno VI (2): 441-475.

Lijphart, Arend. 2000. Modelos de democracia. Formas de gobierno y resultados en treinta y seis países. Barcelona: Editorial Ariel S.A.

Linz, Juan J. 1990a. “The Perils of Presidentialism". Journal of Democracy 1 (1): 51-69.

Linz, Juan J. 1990b. “The Virtues of Parliamentarianism”. Journal of Democracy 1 (4): 84-91.

Linz, Juan J. 1994. "Presidential or Parliamentary Democracy: Does it Make a Difference?". En: Linz, Juan J. y Valenzuela, Arturo (eds.). The Failure of Presidential Democracy. Comparative Perspectives. Baltimore: The Johns Hopkins University Press, vol. 1: 3-87.

Mustapic, Ana María. Enero-marzo 2000. “Oficialistas y diputados": las relaciones Ejecutivo-Legislativo en la Argentina". Desarrollo Económico 156 (39): 571-595.

Nolte, Detlef. 2006 (por publicar). “El presidencialismo chileno después de 1989: lecturas divergentes,". En: Manuel Alcántara Sáez/Leticia M. Ruiz Rodríguez (eds.), Chile: balance de su consolidación democrática. Salamanca: Ediciones Universidad Salamanca.

Palermo, Vicente. 2000. “¿Cómo se gobierna Brasil? El debate sobre instituciones políticas y gestión de gobierno". Desarrollo Económico 159 (40), octubre-diciembre.

Rose, Richard. 1978. What is Governing? Englewood Cliffs: Prentice-Hall, Inc.

Shugart, Matthew Soberg y Carey, John M. 1992. Presidents and Assemblies. Constitutional Design and Electoral Dynamics. Cambridge: Cambridge University Press. 
Schmidt, Manfred G. 2003. Political Institutions in the Federal Republic of Germany Oxford: Oxford University Press.

Schmitter, Philippe C. 1999. “Critical Reflections of the 'Functions' of Political Parties and their Performance in Neo-Democracies". En: Wolfgang Merkel y Andreas Busch (eds.). Demokratie in Ost und West. Frankfurt a.M., Suhrkamp: 475-495.

Valenzuela, Arturo. 1985 (Otoño). “Orígenes y características del sistema de partidos políticos en Chile: proposición para un gobierno parlamentario". Estudios Públicos 18: 88-154.

Valenzuela, Arturo. 1994. "Party Politics and the Crisis of Presidentialism in Chile: A Proposal for a Parliamentary Form of Government". En: Linz, Juan J. y Valenzuela, Arturo (eds.). The Failure of Presidential Democracy. The case of Latin America. Baltimore: The Johns Hopkins University Press, vol. 2: 91-150.

Fecha de recepción: Noviembre de 2005.

Fecha de publicación: Diciembre de 2005. 